\title{
Performance Analysis of CoMP Using Scheduling and Precoding Techniques in the Heterogeneous Network
}

\author{
Myeonghun Chu, Sangmi Moon, Hun Choe, and Intae Hwang
}

\begin{abstract}
Coordinated Multi-Point (CoMP) is considered as a technology in the 3GPP Long Term Evolution-Advanced (LTE-A) system. In this paper, we design and analyze the performance of the Coordinated Beamforming (CB) technique, which is one major category of CoMP. We perform Monte Carlo simulations with a Heterogeneous Network (HetNet) in LTE-A, and confirm the performance through a graph of the Cumulative Distribution Function (CDF). From the simulation results, we show significant performance gain with the CoMP technique, and better performance when we apply various schemes of scheduling and precoding.
\end{abstract}

Index Terms-CoMP, LTE-A, HetNet, scheduling, precoding.

\section{INTRODUCTION}

Mobile communication technology has been constantly evolving to satisfy the communication market, which requires high-speed support for high-capacity and high-quality. The next generation of wireless communication requires a data rate of $100 \mathrm{Mbps}$ class for dynamic user equipment (UE), and 1 Gbps class for static UE. It aims to enable high-speed network access using information devices anytime, anywhere, through integration of wired and wireless communications and broadcasting [1], [2].

Recently, through the prevalence of smart phones, the needs of users for quality anytime, anywhere data services have increased rapidly. Techniques that can support high data rates to users located within cell edges, as well as cell centers, came into demand. A cell center can increase data transmission speed, simply by using the support of additional antenna ports for each cell. But in the case of a cell edge, it is difficult to increase the data rate up to any limitations without cooperation, because this location receives too much interference from adjacent cells. Also frequency reuse techniques being deployed using small cells, such as femto cell or pico cell, within the macro cell area can provide high speed data services to dense user areas. Accordingly, the need for efficient methods to control the interference between transfer points is increasing [3].

Current standards and academic issues, such as how to control the interference from point-to-point transmission naming, identify Coordinated Multi-Point (CoMP), which was selected as a work item for Long Term Evolution-Advanced (LTE-A) Release 11.

Manuscript received October 15, 2015; revised March 23, 2016

The authors are with the School of Electronics and Computer Engineering, Chonnam National University, Gwangju, South Korea (e-mail: diqhq12@naver.com,msm0804@naver.com, chnss8812@naver.com, and hit@jnu.ac.kr).
In the paper, we will discuss the basic techniques of CoMP, and the effect of CoMP combined with several techniques, through simulation results. As an issue in fourth generation mobile communication, the CoMP environment in this paper is based on LTE-A systems.

The rest of the paper is organized as follows. Section II defines the CoMP basic system model. Section III briefly presents the various scheduling and precoding schemes. After evaluation the performance of the proposed schemes in Section IV, we conclude the paper in Section V.

\section{SYSTEM MODEL}

\section{A. System Model for HetNet}

The Heterogeneous Network (HetNet) is one of the LTE-A system networks. HetNet complying with the LTE-A Release 11 Scenario 4 is discussed as follows [4].

As shown in Fig. 1(a), it is assumed that a macro cell is divided into three sectors. Three adjacent sectors consist of one cell site, and communication is coordinated. In Fig. 1(b), pico cells exist in a macro cell, and communication is coordinated with the macro cell. Users are uniformly and randomly distributed in each macro cell and pico cell [4], [5].

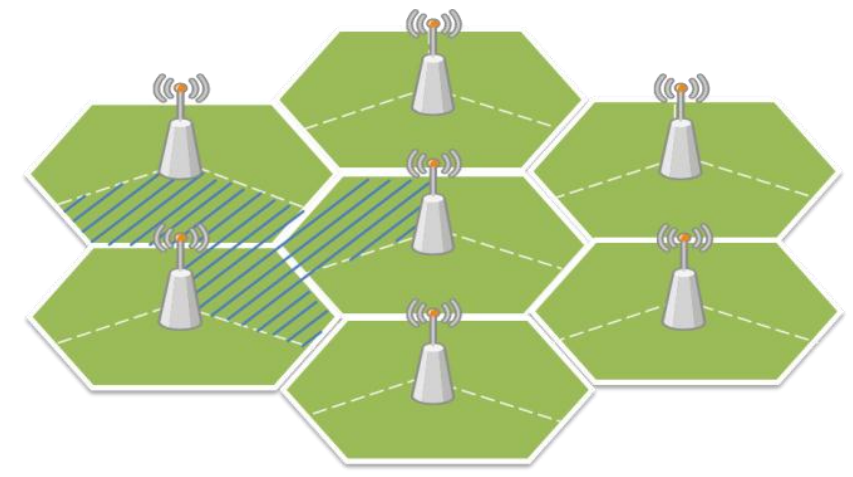

(a)

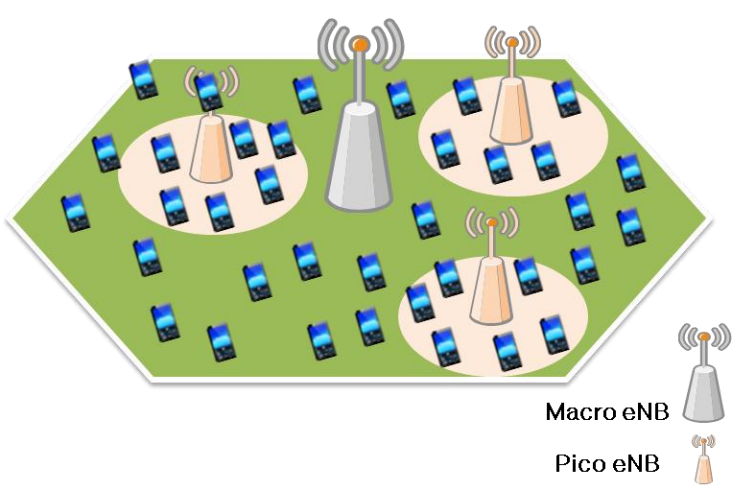

(b)

Fig. 1. Structure of the Heterogeneous Network complying with the LTE-Advanced Release 11 Scenario 4: (a) Macro only, (b) Macro and Pico. 
In the conventional cellular system, users receive a signal from each anchor cell, with interference signals from adjacent cells. We assume that user $M_{1}$ is receiving signals from three cells (denoted as $C_{1}, C_{2}$ and $C_{3}$ ) [6]. Assume $H_{i 1}$ is the channel gain from $C_{i}$ to $M_{1}$. The received signal $Y_{1}$ at $M_{1}$ can be expressed as

$$
Y_{1}=H_{11} W_{1} X_{1}+H_{21} W_{2} X_{2}+H_{31} W_{3} X_{3}+Z_{1}
$$

where, $X_{i}$ is the signal transmitted at $C_{i}, W_{i}$ is the precoding matrix at $C_{i}$, and $Z_{1}$ is the additive white Gaussian noise at $M_{1}$.

As shown in the expression, Inter-Cell Interference (ICI) occurs, and the channel capacity is limited, according to the signal from an adjacent cell being considered as an interference signal. The following expression for $C_{1}$ is located within the user's Signal to Interference plus Noise Ratio (SINR).

If one user receives a signal from its own serving cell, the signals from the other cells affect the user as interference, and then the SINR of M1 can be expressed as

$$
\mathrm{SINR}=\frac{\left\|H_{11} W_{1}\right\|^{2} P_{1}}{\left\|H_{21} W_{2}\right\|^{2} P_{2}+\left\|H_{31} W_{3}\right\|^{2} P_{3}+N}
$$

CoMP technology can control the interference among neighboring cells through cooperative communication technology, which is a promising $4 \mathrm{G}$ cellular standard being developed by the 3GPP standardization group. CoMP can improve the coverage, cell-edge capacity and/or system efficiency. For downlink CoMP, two different approaches are being considered, namely, Joint Processing (JP) and Coordinated Scheduling/Beamforming (CS/CB) in 3GPP LTE-A standard [4].

The SINR for a user that is located within $\mathrm{C}_{1}$ in the CoMP JP scheme can be formulated as

$$
\mathrm{SINR}=\frac{\left\|H_{11} W_{1} \sqrt{P_{1}}+H_{21} W_{2} \sqrt{P_{2}}+H_{31} W_{3} \sqrt{P_{3}}\right\|^{2}}{N}
$$

From the above expression, you can see that the interference signal from the neighboring cells also provides a useful signal through the cooperation among multiple cells, where only noise interferes with the signal.

The received signal at the user within each cell in the CoMP CB scheme can be expressed as

$$
\begin{aligned}
& Y_{1}=H_{11} W_{1} X_{1}+H_{21} W_{2} X_{2}+H_{31} W_{3} X_{3}+Z_{1} \\
& Y_{2}=H_{12} W_{1} X_{1}+H_{22} W_{2} X_{2}+H_{32} W_{3} X_{3}+Z_{2} \\
& Y_{3}=H_{13} W_{1} X_{1}+H_{23} W_{2} X_{2}+H_{33} W_{3} X_{3}+Z_{3}
\end{aligned}
$$

We measure the SINR for each cell by the above expressions, by the precoding matrix that has the highest SINR applied to each cell. As a result, the interference coming from the surrounding cell is minimized, and the received signal strength can be maximized. In this case, the precoding matrix is expressed as follows.

$$
\begin{aligned}
& W_{1}{ }^{\prime}=\operatorname{argmax}_{W_{1}} \frac{\left\|H_{11} W_{1}\right\|^{2} P_{1}}{\left\|H_{21} W_{2}\right\|^{2} P_{2}+\left\|H_{31} W_{3}\right\|^{2} P_{3}+N}(7) \\
& W_{2}{ }^{\prime}=\operatorname{argmax}_{W_{2}} \frac{\left\|H_{22} W_{2}\right\|^{2} P_{2}}{\left\|H_{12} W_{1}\right\|^{2} P_{1}+\left\|H_{32} W_{3}\right\|^{2} P_{3}+N}(8) \\
& W_{3}{ }^{\prime}=\operatorname{argmax}_{W_{3}} \frac{\left\|H_{33} W_{3}\right\|^{2} P_{3}}{\left\|H_{13} W_{1}\right\|^{2} P_{1}+\left\|H_{23} W_{2}\right\|^{2} P_{2}+N}
\end{aligned}
$$

We perform simulations using CoMP $\mathrm{CB}$, which shows a tradeoff between performance and complexity, and which is the best parameter, compared with other schemes.

\section{SCHEdULING AND PRECODING SCHEMES}

In this section, scheduling and precoding schemes are proposed as a way to improve the performance of CoMP. Each technique is described below.

\section{A. Scheduling}

We suggest a scheduling scheme to improve the CoMP performance through using scheduling, and Flexible Frequency Allocation Plan (FFAP) and Coordinated Scheduling (CS) techniques are used.

The FFAP scheme suggests that the whole frequency band be divided into two parts: a CoMP frequency zone for the cell-edge user (CEU)'s transmission, and a single sector frequency band for the cell-center user (CCU)'s transmission, as shown in Fig. 2.

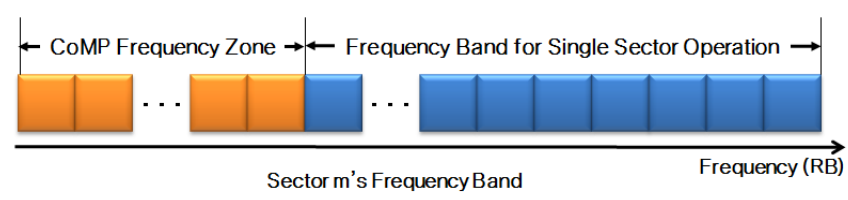

Fig. 2. Structure of the CoMP FFAP scheme.

The differentiation between CEUs and CCUs can be made based on the received SINR at the UE.

$$
\mathrm{SINR} \leq \gamma
$$

where $\gamma$ is a predetermined threshold in $\mathrm{dB}$. The CS scheme is a way in which all users that exist in one cell can be distinguished into CCU or CEU and then, the sum of highest priority of CCU for each sector in one cell and the highest priority of CEU in one cell are compared. If the CEU's priority is higher, the system will be calculated based on the CoMP mode. Otherwise, CCUs are calculated based on the Non-CoMP mode. The flow chart for the CS scheme is shown in Fig. 3. 


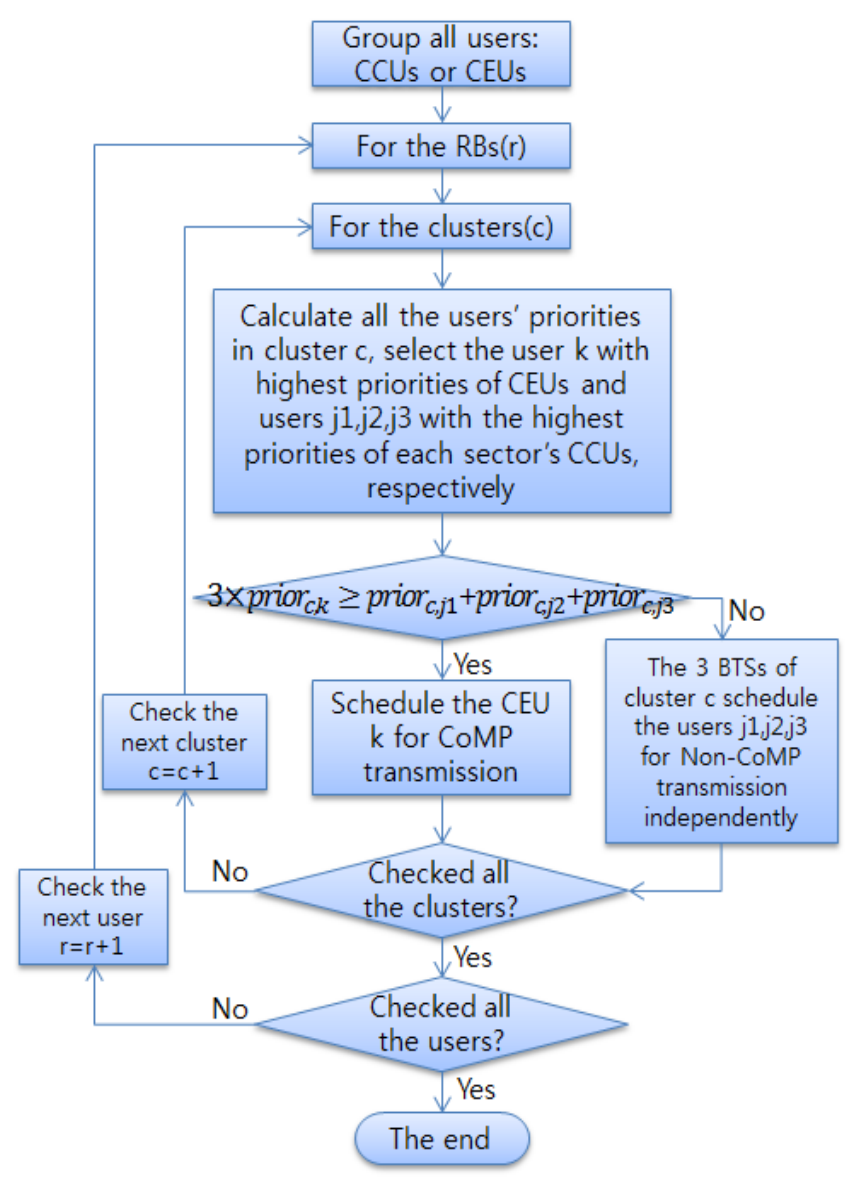

Fig. 3. Flow chart of the CoMP CS scheme.

\section{B. Precoding}

A precoding scheme is another way to improve the performance of CoMP. Precoding is a technique to increase the SINR and spectrum efficiency of a signal, by multiplying a specific matrix with the channel in the transmitter. Singular Value Decomposition (SVD), Polar Decomposition (PD), Tomlinson Harashima Precoding (THP) and QR Decomposition (QRD) techniques are used as precoding schemes.

Using the SVD scheme, the channel is separated in parallel, by multiplying an orthogonal matrix $U$ and $V$. The basic equation for the channel matrix $H$ and the precoding matrix $W$ are expressed as

$$
\begin{gathered}
H=U \Sigma V^{H} \\
W=V
\end{gathered}
$$

Using the PD scheme, the channel is separated, based on the SVD scheme. The channel matrix $H$ and the precoding matrix $W$ are expressed as follows.

$$
\begin{gathered}
H=Q S P^{H}=A U^{H} \\
A=Q S Q^{H} \\
W=U=P Q^{H}
\end{gathered}
$$

The THP scheme is a nonlinear precoding based on Costa's "writing on dirty paper result" information theory [7]. In other words, if the transmitter knows the interference signal beforehand, it can get the same channel capacity as the non-interference channel condition. THP precoding additionally uses modulo operation, which is symmetric nonlinear operation based on costa's precoding. The modulo operation is given as

$$
\bmod _{A}(x)=x-A\left(x-\frac{A}{2}\right) / A
$$

A block diagram of this technique is shown in Fig. 4.

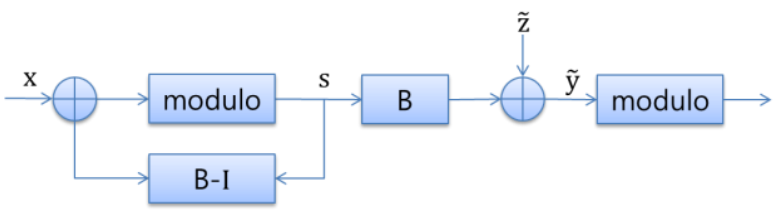

Fig. 4. Block Diagram of THP precoding scheme.

QRD technique creates the precoding matrix through QR decomposition, which factorizes the channel matrix. The basic equations for the channel matrix $H$ and the precoding matrix $W$ are given as

$$
H=R Q^{H}
$$

$$
W=Q F
$$

\section{SimUlation ENVIRONMENTS AND RESUlTS}

We confirm the performance gain according to the use of CoMP technique in heterogeneous network scenario in LTE-A system. In addition, the performance gain for the scheduling and precoding with CoMP techniques will be confirmed. We use Cumulative Distribution Function (CDF) to analyze the SINR and spectrum efficiency.

\section{A. Simulation Environments}

The simulation environment follows the 3GPP LTE-A standard [8]. We perform system-level simulations using Matlab, based on the parameters of Table I. We assume that three pico cells exist within one macro cell.

TABLE I: SIMULATION PARAMETERS

\begin{tabular}{|l|l|}
\hline Parameter & Value \\
\hline Carrier Frequency & $2 \mathrm{GHz}$ \\
\hline Bandwidth & $20 \mathrm{MHz}$ \\
\hline Cellular Structure & $\begin{array}{l}\text { Hexagonal grid, 2-tiers, 7 cell } \\
\text { sites, 3 sectors per cell site }\end{array}$ \\
\hline No. of MS per cell & $100 \mathrm{MSs}$ \\
\hline Antenna Configuration & BS: 2, MS: 2 \\
\hline BS Max TX Power & $49 \mathrm{dBm}-20 \mathrm{MHz}$ Carrier \\
\hline Cell Radius (R) $=$ ISD/ROOT3 & $\begin{array}{l}\text { [Case } 1] \text { Interference limited Micro } \\
\text { Cell }\end{array}$ \\
\hline Path Loss Model & $\begin{array}{l}L=128.1+37.6 l o g 10(\mathrm{R}), \\
\mathrm{R} \text { in kilometers }\end{array}$ \\
\hline
\end{tabular}




\begin{tabular}{|l|l|}
\hline Shadow Std. Deviation & $8 \mathrm{~dB}$ \\
\hline MS Noise Level & $174 \mathrm{dBm} / \mathrm{Hz}$ \\
\hline UE Noise Figure & $9 \mathrm{~dB}$ \\
\hline NodeB Noise Figure & $5 \mathrm{~dB}$ \\
\hline $\begin{array}{l}\text { Correlation distance of } \\
\text { Shadowing }\end{array}$ & $50 \mathrm{~m}$ \\
\hline $\begin{array}{l}\text { Shadowing correlation } \\
\text { between cells/sectors }\end{array}$ & $0.5 / 1.0$ \\
\hline $\begin{array}{l}\text { Minimum distance between } \\
\text { UE and cell }\end{array}$ & $>=35$ meters \\
\hline $\begin{array}{l}\text { BS antenna gain plus cable } \\
\text { loss }\end{array}$ & $\begin{array}{l}14 \mathrm{dBi} \text { for micro, macro cell } \\
\text { case }\end{array}$ \\
\hline Antenna Pattern & $\begin{array}{l}70 \text { degree sectored beam }=70 \\
\text { degree, } A_{m}=20 \mathrm{~dB}\end{array}$ \\
\hline Scheduling scheme & FFAP, CS \\
\hline Precoding scheme & SVD, PD, THP, QRD \\
$*$ ISD = Inter Site Distance & \\
\hline
\end{tabular}

\section{B. Simulation Results}

Using the common transmission (Non-CoMP) method as a baseline for evaluating the performance of the proposed scheme, we send a signal to users.

\section{1) CoMP simulation with scheduling}

The CDF graphs of the SINR are shown when we apply two kinds of scheduling schemes to CoMP CB, in Fig. 5 and Fig. 6, respectively. Fig. 5 is for a Macro UE, which is for a macro cell when communication is coordinated among macro cells. Fig. 6 is for a Pico UE, which if for a pico cell when communication is coordinated among macro cell and pico cell. From these figures, we can find that using the scheduling with CoMP CB brings a performance gain, compared with the non-CoMP. CS with CoMP CB can be seen to provide the highest contribution towards improving the performance.

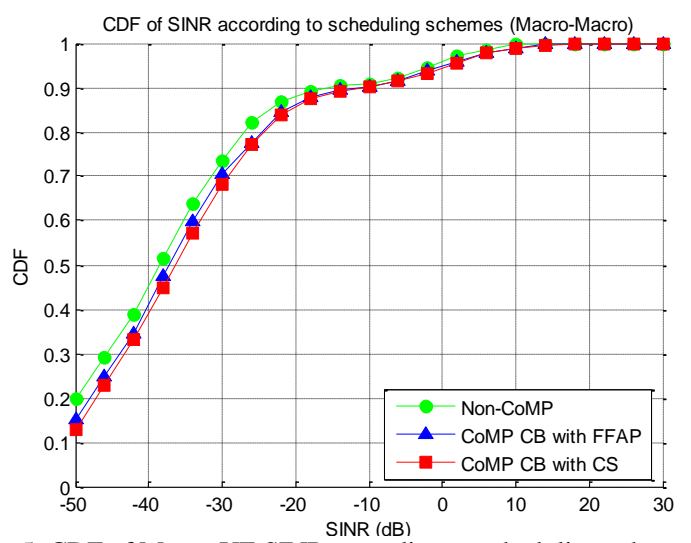

Fig. 5. CDF of Macro UE SINR according to scheduling schemes.

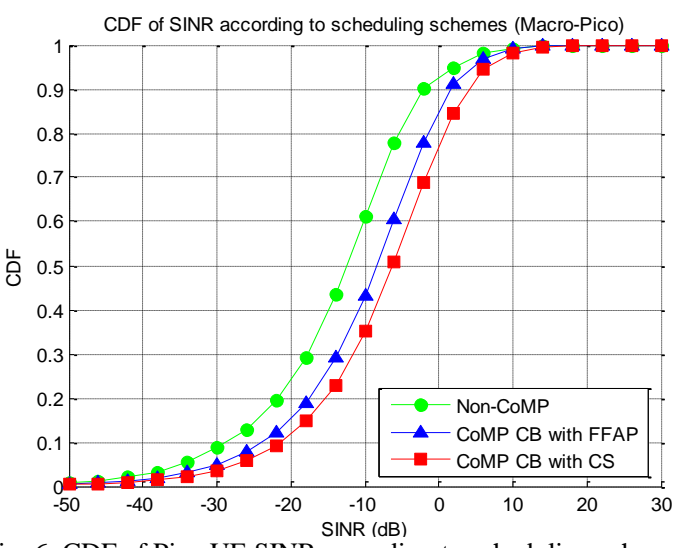

Fig. 6. CDF of Pico UE SINR according to scheduling schemes.
The CDF graphs of spectrum efficiency are shown when we apply two kinds of scheduling schemes to CoMP CB as in Fig. 7 and Fig. 8, respectively. Fig. 7 is for Macro UE, when communication is coordinated among macro cells. Fig. 8 is for Pico UE when communication is coordinated among macro cell and pico cell. From these figures, we can find that using the scheduling with CoMP CB brings a performance gain, compared to with non-CoMP. CS with CoMP CB can be seen to offer the highest contribution to improve the performance.

Also, the performance of the Pico UE is better than the Macro UE case.

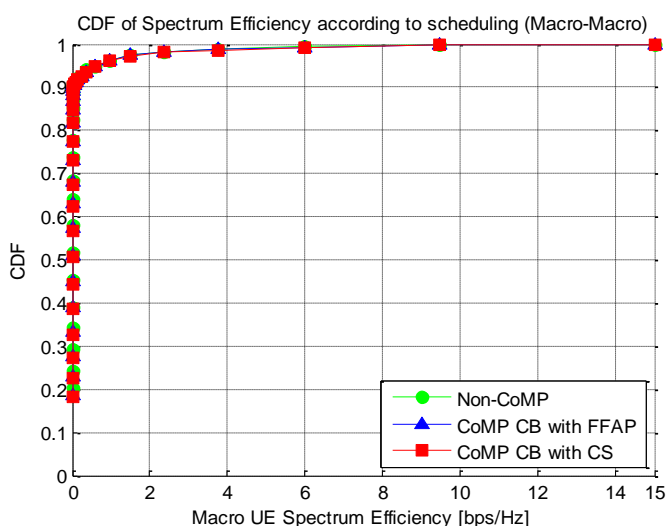

Fig. 7. CDF of Macro UE Spectrum Efficiency according to scheduling schemes.

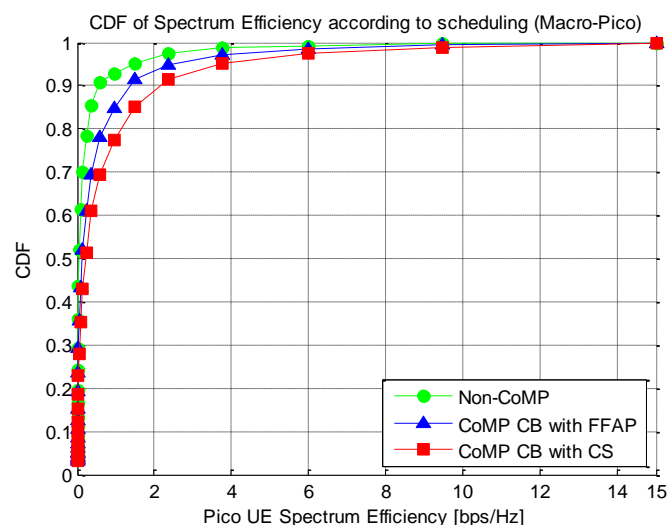

Fig. 8. CDF of Pico UE Spectrum Efficiency according to scheduling schemes.

\section{2) CoMP Simulation with precoding}

Here, the CDF graphs of SINR are shown when we apply various precoding schemes to CoMP CB, in Fig. 9 and Fig. 10, respectively. Fig. 9 is for Macro UE, when communication is coordinated among macro cells. Fig. 10 is for Pico UE, when communication is coordinated among macro cell and pico cell. From these figures, we can find that using SVD precoding with $\mathrm{CB} / \mathrm{CS}$ brings a performance gain, compared to PD, THP and QRD schemes.

The CDF graphs of spectrum efficiency are shown when we apply various precoding schemes to CoMP CB, in Fig. 11 and Fig. 12, respectively. Fig. 11 is for Macro UE, when communication is coordinated among macro cells. Fig. 12 is for Pico UE when communication is coordinated among macro cell and pico cell. From these figures, we can find that using SVD precoding with CB/CS brings a performance gain, compared to PD, THP and QRD schemes.

Also, in the case of Pico UE, the overall performance is better than in the case of Macro UE, because the pico cell is 
located at the edge of the macro cell.

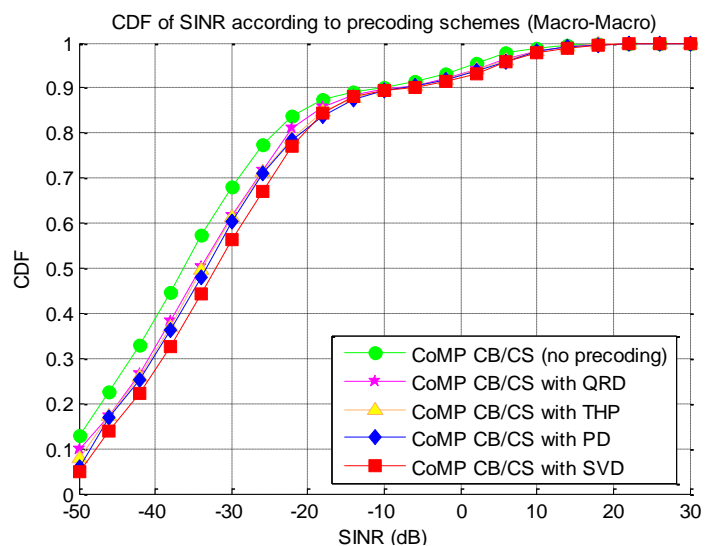

Fig. 9. CDF of Macro UE SINR according to precoding schemes.

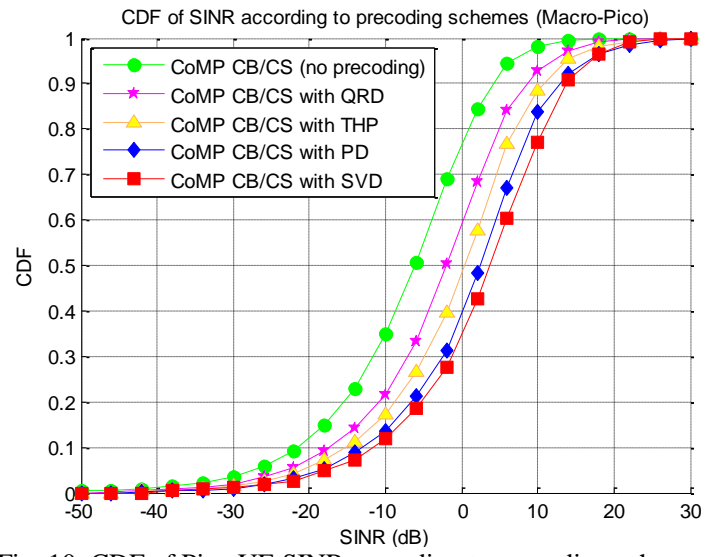

Fig. 10. CDF of Pico UE SINR according to precoding schemes.

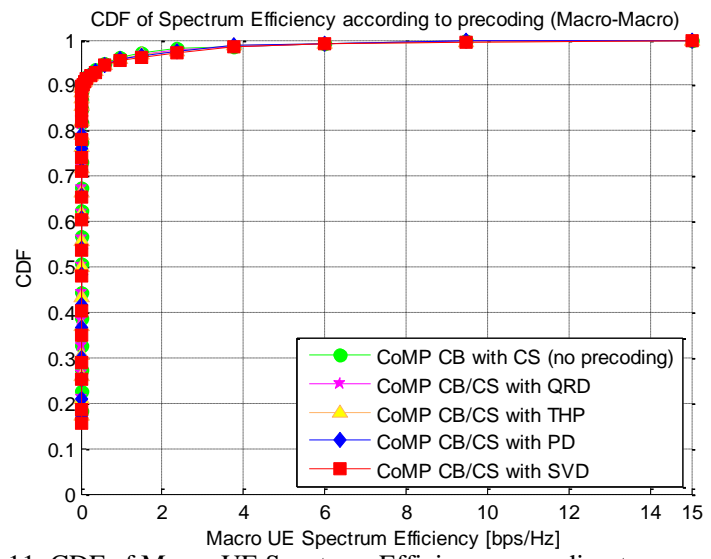

Fig. 11. CDF of Macro UE Spectrum Efficiency according to precoding schemes.

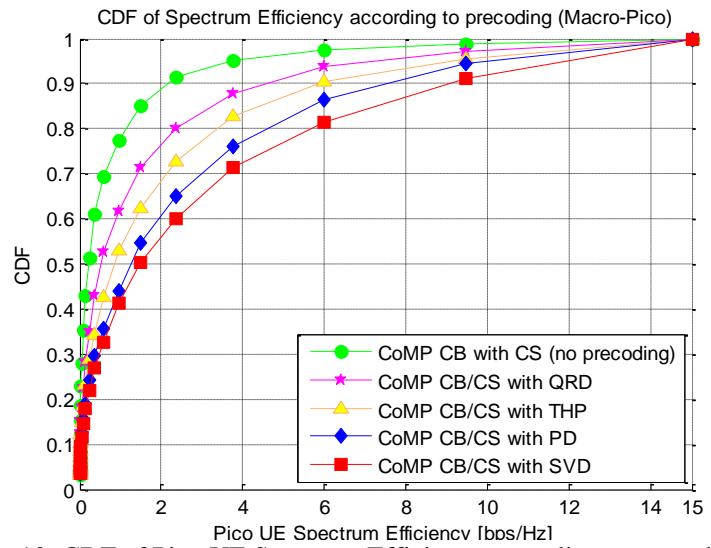

Fig. 12. CDF of Pico UE Spectrum Efficiency according to precoding schemes.

\section{CONCLUSIONS}

We demonstrate a performance analysis of CoMP technique, for multi-point cooperation among users for next-generation cellular systems. System-level simulation results are based on HetNet in the LTE-A system.

We compare and analyze the performance according to the CoMP technique applied, in two cases; one is among only macro cells, the other is among macro cell and pico cell. In both cases, we can get an improved performance when using CoMP technique. It is also shown that CoMP techniques combined with scheduling and precoding schemes achieved further improvement of performance.

As a result, we confirm that CoMP technology can be applied to HetNet which has pico cells within a macro cell in the LTE-A system.

\section{ACKNOWLEDGMENTS}

This research was supported by the MKE (The Ministry of Knowledge Economy), Korea, under the ITRC(Information Technology Research Center) support program (NIPA-2013-H0301-13-3005) supervised by the NIPA(National IT Industry Promotion Agency). This study was financially supported by Chonnam National University, 2012.

\section{REFERENCES}

[1] 3GPP TR 36.913, Requirements for Further Advancements for E-UTRA (LTE-Advanced), 3rd Generation Partnership Project.

[2] 3GPP, "IMT advanced technical requirements - An India perspective," REV-080050, Shenzhen, China, April 7-8, 2008.

[3] Samsung, "Standard trends and performance analysis of cooperative communication on a point-to-point transmission based on LTE-A," TTA Journal, vol. 139, pp. 94-99, 2012.

[4] 3GPP TR 36.819, Coordinated Multi-Point operation for LTE Physical Layer Aspects (Release 11), 3rd Generation Partnership Project, Sept. 2011.

[5] Y.-P. Zhang, "Joint transmission for LTE-advanced systems with non-full buffer traffic," in Proc. Vehicular Technology Conference, 2012, pp. 1-6.

[6] Y.-H. Nam, "Cooperative communication technologies for LTE-advanced," in Proc. ICASSP, 2010, IEEE, pp. 5610-5613.

[7] M. H. M. Costa, "Writing on dirty paper," IEEE Trans. Info. Theory, vol. 29, no. 3, pp. 439-441, May 1983.

[8] 3GPP TR 36.814, Further Advancements for E-UTRA Physical Layer Aspects(Release 9), 3rd Generation Partnership Project.

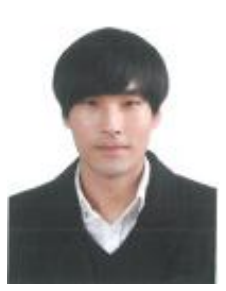

Meonghun Chu received a B.S. degree in electronics and computer engineering from Chonnam National University, Gwangju, Korea in 2015. He is currently a master's student in the School of Electronics \& Computer Engineering at Chonnam National University, Gwangju, Korea from 2015. His research interests are OFDM, cooperative communications, relay communications, massive MIMO and next generation wireless communication systems.

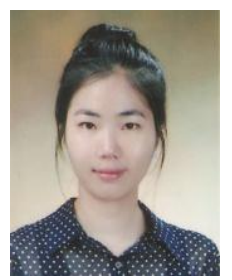

Sangmi Moon received her B.S. and M.S. degree in Electronics \& Computer Engineering from Chonnam National University, Gwangju, Korea, in 2012 and 2014, respectively. She is currently a Ph.D. student in the School of Electronics \& Computer Engineering at Chonnam National University, Gwangju, Korea since 2014. Her research interests include ICIM, D2D, small cell enhancement and next generation wireless communication systems. 


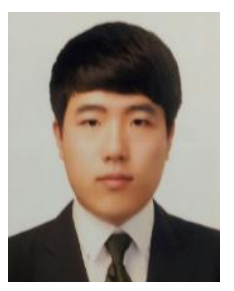

Hun Choe received a B.S. degree in electronics \& computer engineering from Chonnam National University, Gwangju, Korea in 2015. He is currently a master's student in the School of Electronics \& Computer Engineering at Chonnam National University, Gwangju, Korea from 2015. His research interests include mobile and next generation wireless communication systems, MIMO, OFDM and CoMP.

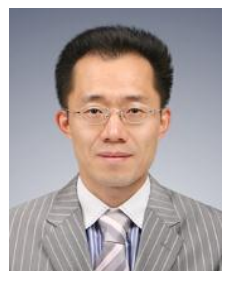

Intae Hwang received a B.S. degree in electronics engineering from Chonnam National University, Gwangju, Korea in 1990 and a M.S. degree in electronics engineering from Yonsei University, Seoul, Korea in 1992, and a Ph.D. degree in electrical \& electronics engineering from Yonsei University, Seoul, Korea in 2004. He was a senior engineer at LG Electronics from 1992 to 2005 . He is currently a professor in the School of Electronics \& Computer Engineering at Chonnam National University, Gwangju, Korea from 2006. His current research activities are in digital \& wireless communication systems, mobile terminal system for next generation applications, physical layer software for mobile terminals, efficient algorithms for MIMO, OFDM, MIMO-OFDM, Relay, ICIM, CoMP, D2D, SCE, and MTC schemes for wireless communication. 